\title{
Neuere Ansichten über Entstehung und Verhütung der Tuberkulose.
}

\author{
Von \\ Dr. Blümel, Halle a. S., \\ Spezialarzt für Lungen- und Halskrankheiten, ärztlicher Leiter der Fürsorgestelle "Halle“ \\ (Assistent in Brobmers Anstalt 1904/05).
}

30 Jahre wissen wir jetzt schon, dass die Tuberkulose durch den Kochschen Bazillus erzeugt wird. Auch Brehmer erlebte. noch diese grundlegende Entdeckung, die ihm allerdings in sein Gebäude, das er sich aus jahrzehntelanger Erfahrung aufgebaut hatte, nicht passen wollte. Und auch heute $h a b e n$ die vom Altmeister Brehmer vertretenen Begriffeder Vererbung, der Belastung und der Disposition, trotz der Entdeckung des Bazillus, noch neben dem Bazillus ihre Berechtigung. Auch in der neuesten Zeit ist man sich zumeist darüber einig, dass z u r Ansteckung mit Tuberkulose zwar der Tuberkelbazillus gehört, dass aber dazu, um aus der Ansteckung eine Erkrankung, eine Schwindsucht zu machen, noch mehr gehört, einedurch angeborene oder erworbene Ursachen herbeigeführte Krankheitsbereitschaft. Das erschwert ja gerade den Kampf gegen die Tuberkulose so sehr, dass die Infektion allein nicht die Ursache der Erkrankung ist, sondern so vielerlei Umstände die Mitursachen oder die auslösende Veranlassung bilden. Im nachstehenden soll zu den letzten Arbeiten über Entstehung der Tuberkulose Stellung genommen werden. 


\section{I. Ätiologie der Tuberkulose.}

\section{Typus humanus oder bovinus?}

Dass die Tuberkulose durch den Tuberkelbazillus erregt wird, wird nach Kochs Entdeckung nicht mehr bestritten. Aber der sogenanntc modifizierte Kochsche Standpunkt, dass die gefährlichste Infektionsquelle der lungenkranke Mensch sei, demgegenüber die Rindertuberkulose sehr in den Hintergrund trete, findet noch manchen Gegner. Es ist vor allem die Orth sche Schule, die sich in einem gewissen Gegensatz zu der vom $\mathrm{K}$ a is erlichen Gesundheitsa $\mathrm{m} t$ vertretenen $\mathrm{Koch}$ schen Anschauung stellt. Nach Orth (1) sind in 10\% aller Kindertuberkulosen Perlsucht. bazillen die Erreger, nicht nur was die leichteren örtlichen, sondern auch die schwereren und sogar generalisierten, ja zum Tode führenden Erkrankungen betrifft. Ja, es ist noch weiter damit zu rechnen, dass infolge einer Umwandlung der Bazillen anscheinend humane im Grunde doch auf bovine zurückzuführen sind. Damit wäre die Gefahr der Rinderbazillen eine noch viel grrössere. Und wenn man noch hinzunimmt, dass eine infantile bovine Infektion es mitverschulden kann, wenn sich später infolge einer Neuinfektion eine Lungenschwindsucht entwickelt, so ist $0 \mathrm{rths}$ Forderung wohl verständlich, den Kampf gegen die Rindertuberkulose nicht zu vernachlässigen.

W eber $(2,3)$ glaubt nun nicht, dass die $\mathrm{Cm}$ wand $\mathrm{lung}$ des Typus humanus in den Typus bovinus wirklich ein häufiges Vorkommnis ist. So sah er einen Knaben, bei dem während eines $10^{1} / 2$ jährigen Aufenthaltes des Tuberkelbazillus im Körper sich der Typus bovinus erhalten hat.

Aus der Orth schen Schule bringen $\mathrm{Dammann}$ und $\mathrm{R}$ abinowitsch (4) neue Unterlagen bei, nach denen bei 290 des untersuchten Kindermaterials sich der Typus bovinus fand. Allerdings handelt es sich nur um Drüsen-, Bauchund Darmtuberkulose. Die Autoren wollten damit teilweise Fra s o r s (Edinburg) Ansicht bestätigen, der in $61,20_{0}$ seiner Fälle bovine Bazillen nachgewiesen haben will.

Das wäre eine ungeheure Zahl, die auch von anderen deutschen Autoren ganz entschieden bezweifelt wird. So weist Möller (5) an einem doppelt so grossen Material (12 eigene und 151. Fälle anderer Autoren) nach, dass Rinderbazillen nur in $2,45 \%$, der Fälle die Krankheitsursache waren. A u ch K n o c h e n - 
und Gelenktuberkulose wird zumeist durch den Typus humanus hervorgerufen.

$\mathrm{Zu}$ etwas höheren Zahlen $(6-80 ; 0)$ kommt Besche (6). Er fand in $\mathrm{Christiania} \mathrm{bei} 50$ Fällen von Kindertuberkulose 45 mal den Typus humanus, 3 mal sicher den Typus bovinus und 2 mal atypische Formen.

Die Zahlen sind wohl sicher zu hoch gegriffen, und die sehr sorgfältig und kritisch gewonnenen des Kaiserlichen Gesundheitsamtes kommen wohl der Wirklichkeit am nächsten. Weber $(2,3)$ weist noch besonders darauf hin, dass die Rindertuberkulose unmöglich einen so grossen Einfluss haben könne. Denn nach den statistisch-ethnographischen Betrachtungen besteht kein Unterschied in der Häufigkeit der Menschentuberkulose zwischen den Läudern, wo Rindertuberkulose vorkommt, und solehen, wo sie fehlt. Die bekannte Sammelforschung des Kaiserlichen Gesundheitsamtes ergab auch, dass von 280 Kindern, die alle die Milch eutertuberkulöser Küho getrunken hatten, schon seit dem Säuglingsalter, nur 2 an Tuberkulose crkrankt waren, und während $7 \mathrm{jähriger} \mathrm{Be-}$ obachtung war kein Todesfall an Tuberkulose eingetreten. Die bovine Infektion betrifft überwiegend das Kindesalter und stellt zumeist eine Fütterungstuberkulose dar, die das Abdomen und die Halsdrüsen befällt. Der erwachsene Mensch ist also gegenüber dem Typus bovinusder Tuberkelbazillen nahezu völlig immun, während der jugendliche Organismus ihr Eindringen zwar nicht verhindern $\mathrm{kann}$, aber doch fast immer imstande ist, den Kampf mit den eingedrungenen Bazillen siegreich durchzuführen. Sch mey meint, dass auch bei jugendlichen Menschen die Immunität mit der Zeit eine vollkommene werdell wird. Denn sie wird erst in der Stammesentwickelung der Menschen crworben, wie der Vergleich mit den Affen zeigt, die weder gegen die Tuberkelbazillen bovinen noch gegen die humanen Ursprungs einen Schutz besitzen.

Im übrigen müssen wir, im Hinblick auf die Rinderbazillen, sagen, dass die Milch entschieden eine Gefahr bilden kann; wie Deist (8) nachweist, besonders in kleinen Ortschaften, wo eine grössere Menge Mischmilch fehlt. Aber durch entsprechende Aufklärung der Milchverkäufer, durch eine zweckmässige Milchhygiene einerseits, und durch ein vernünftiges Verhalten der Milchverbraucher, also durch Kochen der Milch andererseits, lässt sich die Gefahr von seiten der Milch ja ganz vermeiden. Viel schwerer ist es - darauf soll später eingegangen werden - , die Hauptquelle der Ansteckung, den kranken Menschen, auszuschalten. 
Die Typentrennung in bovinus und humanus ist am verlässlichsten am Tierversuch möglich, und zwar im subkutanen Kaninchenversuch. Bei Typus bovinus geht das Tier im Laufe von 6 Wochen bis 4 Monaten an schwerster Kachexie und generalisierter Tuberkulose zugrunde, während bei Typus humanus gar keine, oder nur sehr geringfügige, gutartige Lokalprozesse entstehen, die ausheilen.

\section{Angeborene Tuberkulose?}

Dass während des intrauterinen Lebens Krankheitserreger wie Tuberkelbazillen geradenwegs übortragen werden, ist eine durch keine neuen Nachweise erhärtete Annahme. Im Gegenteil, der negative Ausfall der Pirquetschen Probe bei Neugeborenen und in den ersten Lebensmonaten spricht ganz gegen eine solche Entstehung und die dann dauernd zunehmende Zahl der Infektionen ganz dafür, dass die Tuberkulose extrauterin erworben wird. Die Tuberkulose der Pla$\mathrm{z}$ en $\mathrm{ta}$ ist aber in letzter Zeit häufiger festgestellt worden, vor allen von der Schmorlschen Schule. Ob wirklich Tuberkelbazillen in die Organe des Fötus von der Plazenta aus eingeschleppt werden, ist noch zu beweisen. Denn erstens müssen sich die Tuberkelbazillen auch im kindlichen Teile der Plazenta finden; aber auch dann ist eine Infektion des Kindes noch schwer, denn der Stoffwechsel zwischen mütterlichem und kindlichem Kreislauf geschieht ja durch Osmose und Diffusion, nicht durch direkten Übergang des Blutes.

Harbitz (9) nimmt eine Übertragung durch Plazentartuberkulose an in einem Falle, wo Kind und Mutter 4 Wochen nach der Entbindung der Mutter an Tuberkulose starben. Da auch in diesem Falle die räumliche Trennung von Mutter und Kind nicht sofort und dauernd durchgeführt war, braucht die Infektion nicht von der Plazenta auszugehen.

Dass auch ohne eine tuberkulöse Erkrankung der Plazenta Tuberkelbazillen von der Mutter auf das Kind übergehen können, läge wohl im Bereiche der Möglichkeit, wenn während dér Geburt Schädigungen der Chorionzottengefässe eintreten. Diese könnten die Tuberkelbazillen von den intervillösen Räumen in den kindlichen Kreislauf übertreten lassen. Aber auch dafür ist neues Material nicht beigebracht.

Gewöhnlich kommt also das Kind tuberkulosefreizur Welt und infiziert sicherst von aussen. Der Ansicht pflichten auch v. Leube (10) und $\mathrm{Hamburger}$ (11) bei. Die Quelle der Ansteckung ist dann, wie schon geschildert, der 
Mensch, und die Eintrittspforte des Bazillus gewöhnlich die Lunge. Jedenfalls konnte $\mathrm{Ghon}$ bei $95 \%$ der Sektionen kirschkern- bis kirschengrosse Lungenherde mit regionärer Lymphdrüsenschwellung. finden.

\section{Disposition im allgemeinen.}

$\mathrm{Zu}$ der sogenannten ererbten Disposition bringen $\mathrm{Ku}$ th $\mathrm{y}$ (12) und Wolff (13) neue Beiträge. Kuthy nimmt zur Frage der Vererbung des Locus minoris resistentiae Stellung (Turban). Er fand bei Mitgliedern derselben Familie in 710\% der Fälle dieselbe Seite in derselben Form und in derselben z. B. benignen Art erkrankt. Wolff sah Erkrankungen sogar in derselben Zeit erfolgen, d. h. die Tochter erkrankte mit 20 Jahren in derselben Weise, in der die Mutter erkrankt war, als sie auch 20 Jahre zählte. Die Bazillen haften eben dort, wo ein Organ durch fehlerhafte Anlage oder Funktion widerstandslos geworden ist. Die anatomischen und funktionellen Abweichungen von der Norm können vererbt oder erworben sein. Das Wesen der Disposition besteht aus pathologischen, teils anatomischen, teils physiologischen Zuständen, die dem einzelnen Individuum eigen sind oder als Familieneigenschaft oder zeitlich dem Individuum anhaften. Damit wäre in den kurzen, knappen Worten Wolffs das Wesen der Disposition erschöpft.

Im übrigen verschlechtert die erbliche Belastung, wie unsere neueren Untersuchungen ergeben haben, di e Progn o s e durchaus nicht. So berichtet Curschmann $(14,15)$, dass in Friedrichsheim die erblich Belasteten ca. 200;0 ausmachten, aber gegenüber Nichtbelasteten nicht schlechter dastanden. Nach ihm p r ädisponiert zwar die hereditäre Belastung für die Erwerbung der Tuberkulose, fördert aber gleich. zeitig das Weiterschreiten der erworbenen Krankheit nicht. Nein, eher begünstigt sie die Besserung und Heilung. Curschmann nimmt daher an, dass bei den sogenannten Belasteten eine, von den Eltern überkommene, oder auch vielleicht durch öftere kleine, wieder überwundene, Infektionen erworbene immunitätsfördernde Eigenschaft besteht. Andererseits werden allerdings diese Immunitätsbestrebungen des Organismus infolge der er höhten Exposition gelegentlich durch massive Infektionen gestört, die zur manifesten Erkrankung führen.

Die neuesten Arbeiten lassen es mehr dahingestellt, ob der Habitus phthisicus der Ausdruck einer vererbten Anlage oder das 
Zeichen einer erworbenen Infektion ist. Ich möchte der Ansicht Pollaks (16) beipflichten, dass zumeist der $\mathrm{Habitus}$ phthisicus ein Ausdruck der schon in den ersten Lebens. jahren erworbenen Infektion ist.

\section{Disposition der Lungenspitzell.}

Die Lungenspitzen werden durch mechan ischfunktionelle Verhältnisse zur Tuberkulose disponiert. Die mechanische Disposition ist zuerst von Freund, betont, dann von $\mathrm{Hart}$ und Harras, Schmorl, Birch$\mathrm{H}$ irschfeld u. a. bestätigt worden. Ba cmeister $(17,18)$ hat vor allem auf die funktionellen Störungen infolge Veränderungen der oberen Brustapertur hingewiesen. Seine exakten Tersuche an Tieren erbrachten del Beweis, dass für di e hämatogene und a erogene Infektion diespitze besonders disponiert sei. Durch Anomalien der Rippenknorpel, Verengerung der oberen Brustapertur, die auch durch Gewohnheit, Muskelschwäche und schlechte Haltung herbeigeführt werden kann, wird der Lymphabfluss erschwert, und damit dem Tuberkelbazillus die Ansiedelung erleichtert. (Auch Russeinatmung, grosse Rachenwucherungen können die Ursachen für Erschwerung des Lymphabflusses sein.)

B a c meister stellte seine Experimente so an, dass er bei der hämatogenen Entstehung der Spitzentuberkulose zuerst eine Stenosierung des Brustkorbes der Kaninchen herbeiführte - er liess den Thorax in eine Drahtschlinge hineinwachsen - und dann Tuberkelbazillen in die Blutbahn und Bauchhöhle verimpfte. Die Herbeiführung der Infektion auf aerogenem Wrege gelang ihm, wenn er die Tiere erst aspirieren liess und dann den Rippenring verengte.

Serog (19) bestätigt Bacmeisters Erfahrungen durch Sektionsbefunde nach der Richtung, dass mechanisch-funktionelle Verhältnisse die Hauptrolle bei der Disposition zur Tuberkulose spielen, wenn sie stärkere Störungen der Ventilation, Blut- und Lymphzirkulation in den Lungen hervorrufen. Er teilt die Sektionsergebnisse von 2 Fällen mit Aortenaneurysma und Lungentuberkulose mit, in denen an den Stellen des stärksten Druckes -... einmal betraf es den rechten, einmal den linken Bronchus - sich auch die schwersten tuberkulösen Veränderungen fanden, während die anderen Lungenteile fast ganz frei geblieben waren. Er schliesst daraus, dass schlecht genährte Zellenkomplexe ihre Widerstandsfähigkeit verlieren und dass Freund s Theorie, dass Veränderungen des ersten Rippenknorpels zur Tuberkulose disponieren, richtig ist. 
Schulze (20) dagegen tritt der sogenannten Freund schen Lehre entgegen. Nach Freund, $\mathrm{Hart}$ und Harras u. a. soll ja die abnorme Kürze des ersten Rippenknorpels eine frühzeitige schalenförmige Verknöcherung und die längsovale statt der querovalen Form der Thoraxapertur die Entwickelung von Lungentuberkulose verursachen können. (Verbiegungen und Verkümmerungen der Verzweigung des hinteren subapikalen Bronchus $\mathrm{Birch}$. Hirschfeld] und Druck der ersten Rippe auf das Verbreitungsgebiet dieses Bronchus [S c h m or l sche Rinne] vervollständigen noch die anatomische Disposition der Lungenspitzen.) Schulze fand, dass die Verknöcherung desersten Rippenknorpels weniger eine primäre Anomalie als eine Folge der Ruhigstellung der Lunge in $\mathbf{f}$ o $1 \mathrm{~g}$ e einer Erkrankung sei. Die beschriebenen Aperturveränderungen findet man auch bei an anderen Krankheiten Gestorbenen. Sio entstehen als Folge fehlerhafter Körperhaltung, also auch bei Tuberkulösen. Gelenkbildung im ersten Rippenknorpel, die nach den genannten Autoren die Heilung einer Lungentuberkulose begünstigen soll, hat nach $\mathrm{Sch}$ c lze keine festen Beziehungen zur Heilung einer Tuberkulose; sie ist zumeist eine Folge der stärkeren Bewegung im Schultergürtel.

Es ist bemerkenswert, dass von pathologisch-anatomischer Seite diese Feststellungen nach einer $\mathrm{Hart}$ entgegengesetzten Richtung erfolgen. Die praktischen Schlussfolgerungen der Freundschen Lehre in bezug auf Eingriffe bei Lungentuberkulose sird ja nur in sehr wenigen Fällen gezogen worden. Operative Behandlungen, Durchschneidung des ersten Rippenknorpels bei Spitzenerkrankungen, sind vereinzelt geblieben.

Gestützt wird die Fr e und sche Lehre wieder durch eine Arbeit K ü $\mathrm{ch}$ en hoffs (21). Fr untersuchte die Beziehungen des hohlrunden und des runden Rückens zur Entstehung von Lungentuberkulose und kommt zu folgenden Feststellungen: Die leichten kyphotischen Krümmungen des oberen Teiles der Brustwirbelsäule führen zur Beeinträchtigung der Lungenspitzen und zu Schädigungen. Es resultiert eine persistierende $\mathrm{S}$ c h m o r l sche Rinne und die Birch-Hirschfeldsche Verkümmerung des hinteren oberen Spitzenbronchus.

Die tuberkulöse Infektion der Lungen erfolgt durchaus nicht immer in der Spitze; die Spitzentuberkulose zeigt aber sehr oft die Neigung, voran zu schreiten, während die primäre Tuberkulose der übrigen Teile meistens zur Ausheilung neigt. Die Gründe dafür müssen hauptsächlich in mechanischen Verhältnissen liegen. 
Dass generell Verengerungen der oberen Brustapertur zur Tuberkulose disponieren, und weil diese Erscheinungen sich zur Zeit des grössten Wachstums am meisten ausprägen ( $\mathrm{H}$ a r t), die Tuberkulosemorbidität und -mortalität aus diesem Grunde im Pubertätsalter so stark anschwillt, lässt sich nicht mit Sicherheit entscheiden. Dass es aber mechanisch-funktionelle Verhältnisse, Verengerungen der oberen Brustapertur sind, die die Lungen s itze n vor anderen Teilen der L u n g en als Hauptsitz der Tuberkulose erscheinen lassen, ist durch die neuesten Untersuchungen mit Sicherheit erwiesen.

\section{Infektion und Erkrankung.}

Die Ansteckung mit Tuberkulose geschieht sehr leicht, solange der Mensch noch im Kindesalter steht. Je $\mathrm{kle}$ iner das Kind, um so schneller erkrankt es an Tuberkulose. Mit zunehmendem Alter bildet sich eine zunehmende, natürliche Tuberkulosefestigkeit heraus, und die Empfänglichkeit für Tuberkulose sinkt von Jahr zu Jahr. Eine eimmalige Infektion nit 'Tuberkulose bewirkt sogar einen gewissen, wenn auch geringen Schutz. Stärkeren Reinfektionen von aussen oder von wieder aufflammenden Herden des eigenen Körpers hält dieser Schutz oft nicht stand. Die Folge ist dann eine Frkrankung an Tuberkulose, die eintritt, wenn der Nährboden für die Entwickelung des Bazillus günstig ist. [Siehe auch Liebe (22), B löte (23) und vor allem die vielen auf diesem Gebiete bahnbrechenden Arbeiten von $\mathrm{R} \ddot{\mathrm{m}}$ er und $\mathrm{H}$ a $\mathrm{mburger}$.)

Eine langjährige Durchseuchung mit Tuberkulose, wie sie im alten Europa der Fall ist, schafft cine gewisse Widerstandskraft gegen die Krankheit und ist auch die Ursache, dass die Tuberkulose in der Mehrzahl der Fälle nicht akut, sondern in Form der sogenannten chronischen Lungenphthise auftritt. Das geht aus den Arbeiten der Forscher hervor, die sich mit der 'Tuberkulose in anderen Erdteilen befassten, wie Deycke (24) in der Türkei, Heim (25) und Scherer (26) in den deutschen Schutzgebieten. Je jungfräulicher ein Boden ist, desto melnr akute Formen ergeben sich.

Das sehen wir ja auch in der Praxis so oft, und nach dieser Richtung gehen ja auch unsere Feststellungen bezüglich des Schutzes, der durch erbliche Belastung erworben wird. Starke, stets gesunde Männer, deren Familien beiderseits seit Generationen tuberkulosefrei waren, erkranken an einer akut verlaufenden Tuberkulose, wenn zufällig eine Veranlagung 'zu erkranken durch eine zeitweilige Widerstandsherabsetzung des Körpers gegeben ist. Und die sog. geborenen 
„Schwindsuchtskandidaten“ trotzen sogar oft jahrzehntelang der weiteren Ausbreitung ihrer Erkrankung, trotzen überhaupt der Entwickelung einer Erkrankung aus der vorhandenen Infektion.

Wir kennen aber die Widerstandsfähigkeit des einzelnen nicht, wissen nicht, welcher Grad von Infektion gerade noch einen Schutz und welcher schon eine Erkrankung verursacht, und deshalb ist für uns auch die Prophylaxe so schwer. Denn stürbe die Tuberkulose ganz aus, würden wir beim Besuche von Iändern, die 'Tuberkulose noch haben, ihr wie dio Türken in der asiatischen Türkei gegenüberstehen, unseren mühsam errungenen schutz entbehren und akut erkranken.

Aber diese Sorge ist natürlich zurzeit gar nicht vorhanden. Noch ist die Tuberkulose - ich komme darauf im nächsten Kapitel bei der Prophylaxe zurück - eine so verbreitete Krankheit, dass alles darauf ankommen muss, die Ansteckung möglichst zu verhindern. Tuberkulöse Herde (Zeichen stattgehabter Infektion) und damit einen gewissen Schutz vor der Erkrankung an akuter Tuberkulose werden wir in der Mehrzahl ohne unser Wissen und Wollen schon erwerben.

\section{Prophylaxe der Tuberkulose.}

Auf einige Punkte der Verhütung der Tuberkulose ist schon im vorigen Abschnitt eingegangen worden. Es ist wichtig, dass wir uns, bevor wir die Massnahmen der Verhütung selbst im einzelnen besprechen, noch einmal einige statistische Tatsachen ror Augen halten.

\section{Statistische Feststellungen.}

Behla (27) hat die Statistik nach Altersklassen geo r dnet und dabei wichtige Erscheinungen aufgedeckt. Die Gesamtsterblichkeit in Preussen ist bekanntlich seit 1876 von 30,95 auf 14,58 gesunken (auf 10000 Lebende berechnet). Am stärkste n ist aber an dieser Abnahme die höchste Altersklass e beteiligt, die Sterblichkeit ging im Alter über 60 Jahre von 77,62 auf 19,49 zurück (= rund 58). Der Rückgang ist d o p pelt so gross wio der der Altersklasse zwischen 60 und 30 Jahren, nämlich von 48,62 auf 19,49 (= rund 29). In der Altersklasse von 30 bis 15 Jahren sank die Sterblichkeit nur von 26,91 auf 17,48 (also um rund 9,5). Unheimlich gering aber ist das $\mathrm{Ab}$ sinken vom 15. bis 0 . Jahre, nämlich $7,89 \mathrm{zu} 6,74$ (= rund 1,0). Dabei entfällt die grösste Besserung noch auf das 1. bis 5. Jahr 
(11,72 zu 8,22), vom 0. bis 1. Jahr ist das Verhältnis sogar 22,24 zu 18,40 , während in der Altersklasse vom 5 . bis 10. Lebensjahr die Zahl der Todesfälle sogar zugenommen hat, nämlich von 4,17 a uf 4,32. Vom 10. bis zum 15. Jahr sind die Zahlen 5,71 zu 5,21, so dass der Rückgang hier nur 0,5 beträgt.

Für Baden soll übrigens nach D i esel (28) ein weiterer Rückgang der Tuberkulosesterblichkeit auch im Kindesalter stattgefunden haben.

Wenn wir nach den Ursachen fragen, die im Kindesalter noch die Mortalität unverändert erscheinen lassen, so müssen wir sie vor allem in der zu seltenen Asylierung der Schwer-Tuberkulösen suchen [s. Fürst (29)]. Wenn in Berlin 50, in München $30 \%$ der Tuberkulösen in Krankenhäuserı sterben, so haben diese schon bis zu ihrer Aufnahme, die gewöhnlich kurz vor ihrer Auflösung geschieht, ihre Umgebung infiziert. Und damm müssten die Zahlen für alle Städte möglichst $50 \%$ sein und noch höher. Ich fürchte aber, dass sie oft, z. B. auf dem Lande, noch viel geringer sind.

Unsere sozialen Gesetze sind sogar in vielen Fällen of t sehuld daran, dass sich die Tuberkulösen so ungern in Krankenhäuser aufnehmen lassen. Die Schwerkranken, Arbeitsunfähigen beziehen eben Invalidenrente; gehen sie nun in Krankenhäuser oder Pflegeheime, wird ihnen diese gewöhnlich genommen zur Bestreitung der Verpflegungskosten. Der Schwerkranke bleibt daher lieber in der Familie und übernimmt die Pflege der Kinder, während der gesunde Ehegatte dem Verdienst nachgeht. Denn die Rente bildet das feste Einkommen der Familie, den Grundstock zu Miete und Lebensunterhalt. Gegen ihren Verlust wird sich fast überall sehr gesträubt. Und so sehen wir als Folge, dass die Tuberkulosesterblichkeit nicht zurückgehen kann, weil die offenen Tuberkulösen das Reinigen der Zimmer, die Kinderpflege, das Kochen, kurzum den Haushalt besorgen. Dass auch die Anlage unserer allgemeinen Krankenhäuser auf den Tuberkulösen keinen grösseren Reiz ausübt, ist ein weiterer Grund, der die Asylierung hindert. Grosse Krankensäle sind dem chronisch Kranken, lange Bleibenden unangenehm; zudem merkt er, wie er oft nur als notwendiges Übel angesehen wird, als eine Person, die heilbareren Kranken den Platz wegnimmt.

Wie gefährlich für Kinder dies nahe und ständige Zusammensein mit Tuberkulösen ist, dafür einige Zahlen. Oeverland (30) fand bei schulpflichtigen Kindern mit Hilfe der Pirquetschen Probe dort 4 mal so oft ein positives 
Ergebnis, wo Fälle von Tuberkulose in der Familie vorhanden waren. Das Maximum der Infektion (nicht der Erkrankung) zeigte sich auf dem Lande im Alter von 13 Jahren, in der Stadt schon im Alter von 10 Jahren. - Peters (31) fand, dass von erblich belasteten Kindern $71,5^{0}, 0$ die Pirquetsche Reaktion gaben. Er schiebt das mit Recht auf die erleichterte Infektion in der Familie und durch die Wohnung.

In den wohlhabenderen Schichten, wo grössere Räume zur Verfügung stehen, mindestens für jeden Bewohner ein eigenes Bett vorhanden ist, kann der Kranke zumeist auch ohne grosse Schwierigkeiten im Zimmer isoliert werden. Trotzdem fand Kruse (32) bei studenten in $84 \%$ positive Tuberkulinreaktion, ich schliesse aber, dass die Infektionen lange nicht so bösartig sind, wiediein der arbeitenden Bevölkerung. Denn sonst würde die Zahl der Todesfälle auf 10000 Lebende berechnet nicht gar so grosse Unterschiede aufweisen, nämlich 5 bei den Studierenden und 36 auf die ärmeren L e u te. So massive Infektionen, die später zu schweren Neuerkrankungen von innen heraus führen, scheinen in den wohlhabenderen Kreisen, vor allem infolge der weiteren Wohnungen, seltener zu sein. Bei ihnen zeigt die positive Probe eben nur einen leichten Immunschutz an.

Von Dörner (33) wissen wir, aus einer sehr fleissigen Studie, dass in Einzimmerwohnungen Tuberkulosetodesfälle doppelt so häufig sind wie in geräumigeren. Bei offener Tuberkulose der Mutter gehen $33,50^{\circ}$, des Vaters 12,70\% der Kinder an Tuberkulose zugrunde. Wir sehen auch an diesen Fällen wieder, dass die Mutter, die sich naturgemäss mit den Kindern melır befasst, einen viel verderblicheren Einfluss ausübt, wenn sie eine offene Tuberkulose hat. Auch hier wieder spielt die Übertragung von Mensch zu Mensch, die Häufigkeit und Nähe des Verkehrs, die Hauptrolle.

W e in b e r g (34) fand bei seiner Riesenstatistik (5000 Familien mit 18000 Kindern), dass, je näher die Geburtszeit der Kinder dem Todeder Eltern rückt, um so grösser die Sterblichkeit ist. So starben von den im letzten Lebensjahr der tuberkulösen Mutter geborenen Kindern 3/4, von den im letzten Lebensmonat geborenen $90 \%$.

Mit der Schwere der Erkrankung der Eltern nimmt naturoemäss die Produktion der Tuberkelbazillen zu und die Sauberkeit in der Wohnung ab, besonders wenn die Mutter erkrankt ist. - Wie gefährlich die Ansteckungsgefahr von Mensch zu Mensch ist, darauf 
weist auch Engelhardt (35) hin. Er fand auch bei reinlich gehaltenen offenen Tuberkulosen stets Tuberkelbazillen im aspirablen Staub.

Wie schwer schon das früheste Alter bedroht ist, dafür bringt Effler (36) einige Zahlen. Von 58 Säuglingen in Familien mit offener Tuberkulose waren 33 schon im $\mathrm{S}$ ä u gl ing salt e r infiziert.

Über französische Verhältnisse berichtet Ickert (37). Die Mortalität von Kindern, die nicht aus der tuberkulösen Umgebung entfernt wurden, beträgt $60 \%$. Noch grösser ist die Morbidität. Für Kinder, die aus den tuberkulösen Familien herausgenommen und bis zum 13. Lebensjahr bei bäuerlichen Familien erzogen wurden (Oeuvre Grancher) betrug die Mortalität $1 / 2 \%$, die Morbidität $1 / 4 \%$.

\section{Massnahmen zur Verhütung der Tuberkulose.}

Wie schützt sich der einzelne, der nicht in seiner eigenen Familie, in seiner nächsten Umgebung eine Ansteckungsquelle hat? Wir Ärzte sollten uns vielmehr, wie es unsere leider knapp bemessene Zeit ermöglicht, damit beschäftigen, die Widerstandsfähigkeit der Gesunden zu erhalten. Aufklärung in Wort und Schrift, in Versammlungen und der Allgemeinheit zugänglichen Blättern sind zwar sehr nützlich, aber am meisten wirkt der Arzit durch seine Stellungnahme von Fall zu Fall, durch seine persönliche Rücksprache. "Schutz der Gesunden" müsste die Parole heissen. Alles was die Widerstandsfähigkeit der Gesunden erhält und hebt, hilft der Tuberkulose vorbeugen. Dahin gehört (in Form eines Merkblattes):

1. Eine gesunde Wohnung. Sie muss trocken, sonnig und gross genug sein. Kleine, enge, feuchte und lichtlose Räume bedingen eine vermehrte Krankheitsbereitschaft. Wer tagsüber sich in seinen Wohnräumen wenig aufhält, wähle das beste und grösste Zimmer als Schlafraum.

2. Sauberkeit. Die Zimmer sollen feucht aufgewischt und gut abgestäubt werden. Rein ig ung des Körpers, Reinhaltung der Kleidungsstücke, genügend häufiger Wechsel der Wäsche, Mundpflege durch Benutzung der Zahnbürste und saubere Ess- und Trinkgeschirre sind von grosser Wichtigkeit. Zur Sauberkeit gehört auch ein fleissiges L ü f e n d er Z i m m er. Eingeschlossene verbrauchte Luft begünstigt die Entwickelung von Krankheiten, vermindert den Appetit usw. Saubere Betten sind nötig, wenn irgend möglich für jeden ein eigenes. 
3. Zweckmässige Ernährung. Bier und Wein sind keine Nahrungs-, sondern Genussmittel. Als Nahrungsmittel, in grössercr Menge genossen, wirken sie sogar schädlich. Mhr Preis steht in keinem Verhältnis zu ihrem Nährwert. Wer also mit seinen Einnahmen haushalten muss, lege kein Geld für Alkoholika an. II I ch und Kakao sind wesentlich nützlichere Getränke. Sie dienen gleichzeitig der Sättigung und Ernährung. Wem G e mü se in manchen Jahreszeiten zu teuer wird, der erinnere sich, dass Reis, gelbe und grüne Erbsen, Linsen und weisse Bohnen einen ausserordentlich hohen Nährwert haben im Verhältnis zu dem dafür bezahlten Preis. Fle isch ist ein sehr teures Nahrungsmittel. Wo gespart werden muss, ist sein Verbrauch auch ohne Schaden einzuschränken. Käse ist ein billiger Fleischersatz, ebenso Fische. Kartoffeln sind ein billiges Nahrungsm ittel, ebenso Graupen, Griess, Mais, Makkaroni und Nudeln. Besonders wertvoll ist das $\mathrm{Brot}$, jedenfalls viel zweckmässiger als die so viel von Leuten mit gesunden Verdauungsorganen an seiner Stelle genossenen $N$ ährpräparate, wie sie auch heissen mögen, als Sanatogen, Bioson, Biomalz u. a. Der für solche Mittel gezahlte Preis entspricht nicht ihrem Wert für die Ernährung sonst Gesunder, während sie natürlich bei Kranken nach ärztlicher Verordnung schon angezeigt sein können.

Bei seinen Untersuchungen im Landkreise Quedlinburg fand auch Fischer-Def o y (38), dass landwirtschaftliche Arbeit, ein doch sonst als gesund angesprochener Beruf, Gesundheitsschädigungen verursacht, weil die $\mathrm{Sch}$ were der Arbeit in keinem rechten Verhältnis zu der Ernährung stand. Auch Lörner (33) spricht diese Ansicht aus, und F ürbringer (39) bestätigt sie. Wo Kaffee und Bier an die Stelle der trüher getrunkencn Mehlsuppe treten, geht es auf Kosten der Volksgesundheit. Auch dass die Frauenzu wenig kochen können, wird von den genannten Autoren geklagt.

Der Kampf gegenden Alkoholismus isteingutes Unterstützungsmittel im Kampf gegen die Tuberkulose. Mässigkeit und Enthaltsamkeit sind für die Gesunderhaltung des einzelnen ausserordentlich wichtig. So zeigt die neueste Arbeit von Holitscher (40), dass von Heilstät en pfleglingen über $45 \mathrm{Jah}$ ren $2 / 3$ Trinker sind, d. h. solche, die gewohnheitsmässig täglich über 3 Liter Bier oder dieser Alkoholmenge gleiche Mengen Schnaps zu sich nehmen. Da im Alter bis zu 25) Jahren nur 5\% Trinker unter den Heilstättenpfleglingen sind, so geht daraus hervor, dass unheimlich viele Trinker im höheren 
Alter ein Opfer der Tuberkulose werden, weil ihre Widerstandsfähigkeit infolge des übertriebenen Alkoholgenusses sehr abgenommen hat.

4. Trunk und Geschlechtskrankheiten folgen oft aufeinander. Sie untergraben oft die eigene Gesundheit und oft die ganzer Generationen.

5. Eine entsprechende $\mathrm{Ruh}$ e erhält die Widerstandsfähigkeit. Wer tagsüber angestrengt arbeitet, braucht nachts seine $8 \mathrm{St}$ u n d e n $\mathrm{Schl}$ af; Kinder brauchen entsprechend $\mathrm{m}$ e h r. Ausgedehnter Wirtshausbesuch, Zechgelage, Tanzereien verkürzen die notwendige Ruhezeit und schwächen den Körper wie jeder unsolide Lebenswandel. Auch das übermässige Rauchen gehört hierher.

6. Gesunde A r beitsrä u m e und ein ruhiges Arbeiten erhalten die Freude und die Kraft. Wessen Gesundheit durch schädigungen in diesem oder jenem Beruf gefährdet ist, der soll ihn rechtzeitig wechseln.

Kurz möchte ich hier auf die neuesten Feststellungen Dö r n e r s (41) hinweisen, der die Wichtigkeit der wirtschaftlichen Verhältnisse besonders betont. So veränderte sich in einem mehr industriell tätigen Kreise Badens die Tuberkulosesterblichkeit gegenüber einer landwirtschaftlichen Gegend sehr zu ungunsten der ersten. Es ergab sich eine Übersterblichkeit der Frau, vor allem im erwerbsfähigen Alter zwischen 20 und 30 Jahren; sie war $3 \mathrm{mal}$ so gross wie in dem landwirtschaftlichen Bezirk.

Über seine Kraft kann keiner gehen. Soll die Frau Landwirtschaft, Industrie- und Hausarbeit machen, müssen die Angehörigen und sie selber leiden. Bleibt die Wohnung schmutzig, wird nicht hinreichend Sorgfalt auf die Ernährung verwandt, wird die Ruhezeit zu sehr beschränkt, dann steigt die Tuberkulosegefahr, besonders wenn die Arbeit noch ungesund ist, wie in Dörners Fall die Zigarettenmacherei, bei der Muskelkrätte kaum gebraucht werden und zumeist eine gebückte Haltung eingenommen wird.

Andere Massnahmen, die die Tuberkulose verhüten helfen, sind:

7. A blı̈̈rtung. Darunter ist zu verstehen: Abwaschung des Körpers mit kaltem Wasser, Luftbäder im Zimmer oder im Freien; Wasserbäder in Schwimmhallen oder in Flüssen. Gewöhnung an Luft (nicht soviel Stubenhocken!). Ausgedehnter Luftgenuss auf Fusswanderungen an Sonn- und Feiertagen, beim Turnen, Rudern, Schlittschuhlaufen und anderen körperlichen Übungen, Schlafen in kühlen Räumen bei geöffneten Fenstern erhöht die Widerstandsfähigkeit des Körpers. 
8. Gesunde Kleidung. Sie soll nichtzudicht und nicht zu reichlich sein. Ein Unterhemd und ein Unterbeinkleid genügen; für die Frauen ist e in Unterrock, wenn das Beinkleid warm hält, ausreichend. Männer sollen keinen Gürtel, Frauen kein Korsett tragen und natürlich auch keine Rockbänder. Alle Kleidungsstücke sollen von den Schultern herunter entweder an Trägern, die sich auf dem Rücken kreuzen, oder an Unterleibchen getragen werden.

9. Genügende Schonung nach grossen Anstren. gungen des Körpers und nach Krankheiten. Hierhin gehören vor allem Entbindungen, Blutarmut zur Zeit der Entwickelungsjahre, Infektionskrankheiten aller Art, Katarrhe der Luftwege, Stoffwechselkrankheiten usw.

\section{Besondere Massnahmen für Kinder.}

Viel mehr wie bisher sind die Eltern darauf hinzuweisen, dass länger dauernder Husten, häufige Katarrhe der Atmungsorgane, Abnahme des Körpergewichtes, Mattigkeit, Fiebererscheinungen auf Tuberkulose verdächtig sind. Deshalb soll der Schul ar z t nirgends fehlen. Ausserordentlich wertvoll ist seine $\mathrm{Verbindung} \mathrm{mit}$ dem Arzt der Fürsorgestelle. So möchte ich unser Zusammenarbeiten in Halle als besonders erspriesslich hinstellen. Die Schulärzte überweisen alle Kinder, die sie für gefährdet halten oder in Heilanstalten wegen drohender oder bestehender Tuberkulose schicken wollen, dem Fürsorgearzt. Dieser lässt durch seine Schwestern die Häuslichkeit besichtigen, untersucht die Angehörigen und macht dem Schularzt Mitteilung über die der Fürsorgestelle bekannten anamnestischen Angaben der Wohnungs- und Familienverhältnisse. Denn was nützt es, ein Kind aus einer schmutzigen, engen Wohnung in eine Heilstätte zu schicken, wenn es gleich darauf wieder in die alten Verhältnisse zurückkommt? Ich habe als Fürsorgearzt in wiederholten Fällen Veranlassung genommen, erst die häuslichen Verhältnisse zu regeln, um dann auch mit Erfolg Kuren an den Kindern durchführen zu können. Nur so kann man auf Dauererfolge rechnen.

Für Kinder ist natürlich Sauberkeit, sind helle weite Wohnräume, Luftgenuss und zweckmässige Ernährung ein Haupterfordernis. Ist die eigene Wohnung zu eng, kann die Mutter, weil sie selbst mit verdienen muss, sich um die Kinder nicht genügend kümmern, so sind für Säuglinge K r i p p en , für grössere Kinder die B e w a h r anstalten tagsüber in Anspruch zu nehmen. Wo die Wohnungs- 
verhältnisse schlecht sind und die Sauberkeit gering ist, helfen wir uns im Augenblick damit, dass wir die Kinder für die Nacht in Schlafpavillons bringen. Die Kinder bekommen hier Abendbrot und erstes Frühstück, das zweite nehmen sie von hier in die Schule mit, so dass sie nur mittags über zu Hause sind. Der Gedanke, dessen Vorzüglichkeit wir nicht genug preisen können, ist von unserem Stadtarzt Profess or v. Drigalski ausgegangen. Für wenig Widerstandsfähige, nicht gerade kräftige Kinder sind sonst Heil- und Pflegestätten am Ort in Anspruch zu nehmen und ausserhalb Ferienkolonien. Waldluft, besser noch Kuren an der Sec oder in Solbädern haben trotz ihrer nur einige Wochen langen Dauer auf die Gesundung mancher Kinder einen vorzüglichen Einfluss.

$\mathrm{V}$ i el Schaden, viel mehr, als gemeinhin angenommen wird, bringt für die Kinder die Beschäftigung der Mutter a usserhalb des Ha uses. Die Sauberkeit der Wohnung lässt zu wünschen übrig, das Essen, die Abwartung und Erziehung der Kindès. Deshalb sollen die Frauen, auch weil, wie oben erwähnt, die Arbeitsüberhäufung zu Tuberkulose disponiert, nicht wahllos jede Tätigkeit ausserhalb des Hauses übernehmen.

\section{Wie sehiitzt sich die Umgebung, vor allem der Angehïrige von Sehwindsüchtigen vor Ansteckung?}

Die Antwort lautet: am schlechtesten dadurch, dass sie den Kranken fliehen und meiden wie einen Aussätzigen. Arbeitskollegen, die einen Lungenkranken aus Angst vor Ansteckung aus einer Stellung drängen, tun bitter Unrecht. Denn der Kampf gregen die Tuberkulose darf niemals zu einem Kampf gegen die Tuberkulösen ausarten. Der Schutz vor Ansteckung mit Tuberkulose is t überall dort nicht schwierig, wo der Kranke sauber ist und die äusseren Umstände günstig sind. Bedrängt man die Kranken, erreicht man nur, dass sie die Krankheitserschéinungen unterdrücken, ihre Tuberkulose verbergen, und so den Gesunden eine Gefahr werden. Wer sich vor der Benutzung der Spuckflasche entsetzt, erreicht nur, dass der Kranke in sein Taschentuch oder auf den Boden spuckt.

Es muss immer wieder dem Publikum klar gemacht werden, was an dér Tuberkulose ansteckend ist, nämlich die $\mathrm{mit} d \mathrm{em}$ Hustenstoss verspritzten, bazillenhaltigen Tröpfchen und der A uswurf, wenn er so entleert wird, dass er verstäuben kann. Schon aus allgemeinen Anstandsrücksichten hält sich der Hustende die Hand vor den Mund und wendet sein resicht 
ab. Aus demselben Grunde wird er in geschlossenen Räumen nicht auf den Boden spucken. Also eine Gefahr für Mitarbeiter und andere, die nicht gerade mit hustenden Kranken eng zusammen leben, ist nicht vorhanden, wenn sich der Kranke öfter die Hände wäscht und nicht auf den Boden spuckt. Das Publikum legt manchen Dingen einen ansteckenden Charakter bei. Deshalb soll immer wieder darauf hingewiesen werden: Der Schweis $\mathrm{s}$ es $\mathrm{Kranken}$, der seinen Dunstkreis erfüllt, die Luft, die er ausatmet, sind ungefährlich und enthalten keine Ansteckungsstoffe.

Eine $e r h \ddot{h} t$ e Ansteckungsgefahr bedeutet der Kranke für seine Familie, aber auch die kann sich durchaus schützen. Wird der Auswurf nur in ein mit Flüssigkeit gefülltes Gefäss entleert, oder in die täglich zu säubernde Spuchflasche, so bleibt nur noch die Gefahr zu vermeiden, die die mit den Hustenstössen verspritzten Bazillen verursachen. Um ihrem verderblichen Einfluss zu entgehen, dürfen Gesunde nie mit Lungenkranken das B ct t teilen. Jeder Tuberkulöse soll ein eigenes Bett haben. Der Fussboden muss in den von ihm benutzten Zimmern besonders sauber gehalten, die Bettwäsche oft gewechselt werden. Da für Kinder, je jünger sie sind, die Ansteckungsgefahr um so grösser ist, müssen sie a us dem gemeinsamen Schlafzimmer entfernt, ja möglichst auch am Tage fern von dem Kranken gehalten werden. Deshalb ist die beste Vorbeugung zur Verhütung der Ansteckung mit Tuberkulose ein eigenes Zimmer für den Kranken. Tagsüber werden ja grössere Kinder durch den Schulbesuch von der Wohnung ferngehalten, kleinere sollen durch ausgiebigen Aufenthalt im Freien, oder wo die nötige Aufsicht fehlt, durch Aufnahme in Krippen und Bewahranstalten vor zu inniger und langer Berührung mit dem kranken Familienmitglied geschützt werden.

Die Kleidung des Kranken ist besonders sauber zu halten, die Wäsche durch sorgfältiges Kochen und Plätten keimfrei zu machen. Ess-, Trink- und Waschgeschirr soll der Kranke für sich allein benutzen.

Lungenkranke Mütter dürfen Neugeborene nicht stillen, und eigentlich auch nicht pflegen, denn Säuglinge stecken sich am leichtesten an, und ihre Krankheit ist kaum einer Heilung zugänglich. Ebenso ist es zu verwerfen, wenn lungenkranke Männer oder andere Angehörige die Kinder besorgen. Viel,richtiger und zum Schutze der Familie notwendig ist es, wenn man solche Kranken, die nicht den grössten Teil des Tages und die Nacht über von den Kindern getrennt gehalten werden können, zu kinderlosen Familien bringt, in Krankenhäuser oder Pflegeheime gibt, oder wenn sie umher- 
gehen können, recht viel ausserhalb des Hauses hält. Die Kranken sollen sich vor solchen Massnahmen nicht sträuben, nicht überflüssig und leichtfertig ihre Familie gefährden, sondern selbst ein Opfer bringen zum Schutze der Ihrigen. Leider bringt nur zu oft mangelnde Rücksicht des Kranken und falsche Rücksicht der Angehörigen auf die Krankenganze Familien in Lebensgefahr und zum Aussterben.

Vor allem ist die Infektionsmöglichkeit für Kinder unterhalb des 4. Jahres zu vermindern; infizierte Kinder sind vor allem vor Keuchhusten und Masern zu behüten (H a mburger), da nach diesen Krankheiten häufig Tuberkulose ausbricht.

Ausser diesen Vorsichtsmassrahmen ist nötig, dass für Kinder Milch nur abgekocht gegeben wird, um eine Übertragung der Rindertuberkelbazillen zu verhüten, wie schon oben erwähnt.

Fassen wir also noch einmal kurz zusammen, worauf es bei der Verhütung der Tuberkulose ankommt, so ist es: 1. Ge sundheitsmässiges Wohnen und gesundheitliche Lebensführung; 2. Unschädlichmachen des Auswurfs; 3. Verhütung des dichten und dauernden Zusammenlebens in Wohn- und Schlafräumen mit Kranken, besonders in Rücksicht auf die Kinder; 4. Abkochen der Milch.

\section{Die bisherigen Leistungen von Staat und Gemeinde.}

Seit Jahrzehnten sind Staat und Gemeinde bemüht, durch Gesetze und Aufsicht die Gesundheit zu heben und damit dem Schutz vor Tuberkulose zu dienen. Das Krankenversicherungsgesetz ermöglicht es dem kranken Versicherten, rechtzeitig ärztliche Hilfe in Anspruch zu nehmen ohne neue Kosten. Das ist natürlich auch der Tuberkulose zu Nutzen gekommen. Die Ärzte sahen die Kranken früher und stellten die Diagnose schon oft im

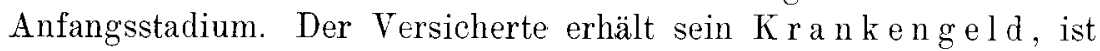
so den augenblicklichen Sorgen enthoben und kann sich Zeit lassen bis zur möglichst weitgehenden Genesung. Die Invaliden vers i ch er ung führte das Werk der Krankenversicherung insofern fort, als sie in ihrem $\S 18$ vor allem der Tuberkulosebekämpfung ein wertvolles Mittel, das $\mathrm{Heilverfahren,} \mathrm{zur} \mathrm{Verfügung} \mathrm{stellte.}$ Heute stehen in Deutschland 147 Volksheilstätten mit 15000 Betten zur Verfügung, in denen jährlich ungefähr 60000 Versicherte für $1 / 4$ Jahr verpflegt werden. Wird auch nur auf 1/4 Jahr die Ansteckungsquelle so aus der Familie entfernt und sie 
nur, was die Cmwandelung offener in geschlossene Tuberkulosen anlangt, zeitweise und auch das nur in einem Teil der Fälle verstopft, so ist doch durch die Heilstätten einerseits ein erheblicher Gewinn an $\mathrm{Nationalvermögen} \mathrm{durch} \mathrm{Wiederherstellung}$ mancher angegriffenen und gefährdeten Gesundheit eingebracht worden. Andererseits haben die Heilstätten durch Anleitung so vieler Tausende von Kranken zu einer vernunftgemässen, gesundheitlichen Lebensweise ausserordentlich viel genützt. Die von ihnen ausgesandten Gesundheitsapostel haben vor allem die Gefahr der Übertragung der Tuberkulose und die Kenntnis, wie sie vermieden werden kann, aufgefasst und in weite Kreise getragen. An und für sich liegt ja eine Bekämpfung der Tuberkulose als Volkskrankheit a u chnicht dem Gedanken der Heilstätten zugrunde. Sie sollen lediglich der Wiederherstellung der Erwerbsfähigkeit dienen, die ja nur den nicht zu Schwerkranken in 3 Monaten zurückgebracht werden kamn. Die Schwer-Tuberkulösen sollen ja von der Heilstättensache gar nicht erfasst werden. Kommen die Heilstätten, was die Verhinderung der Ansteckung mit Tuberkulose und damit die Bekämpfung der Tuberkulose als Volkskrankheit bedeutet, nicht in Betracht, so sind sie für den einzelnen Kranken, der durch sie seine Arbeitsfähigkeit wieder erringt, von ausserordentlicher Bedeutung.

Wo ein Heilverfahren nicht mehr durchführbar ist und die Erwerbsfähigkeit weniger als $2 / 3$ der normalen beträgt, tritt die Invalidenrente ein. Sie ist, wie schon früher erwähnt, das feste Einkommen für den Invaliden und seine Angehörigen. Dass ihre Gewährung gerade den Schwerkranken vom Aufsuchen eines Krankenhauses abhält, ihn also daran hindert, sich selbst als den Bazillenspender durch Entfernung aus der Familie für diese ungefährlich zu machen, lag ja nicht in der Absicht des Gesetzgebers. Wie weil diese Misslichkeit zum Nutzen der Tuberkulosebekämpfung ausgeglichen werden kann, darüber im nächsten Abschnitt.

Die Altersrente tritt ohne Rücksicht auf die Erwerbsfähigkeit im 70. Lebensjahre ein; sie kommt für die Tuberkulosebekämpfung weniger in Betracht, da nur wenige Tuberkulöse des Arbeiterstandes dies Alter erreichen.

Neue Nahrung erhielten die Heilstätten vor 11/2 Jahren durch die neu eingeführte $A \mathrm{ng}$ estell te n versicherung, die die Angestellten bis zu einem Einkommen von 5000 Mark erfasst. Ihre reichen Mittel erlauben es, viel mehr wie bei der Arbeiterversicherung persönlich von Fall zu Fall zu verfahren und das Heilverfahren nach Bedarf auf $1 / 2$ Jahr und darüber auszudehnen. 
Auch die neu in Kraft getretene $W$ itwen- $u n d W$ a isenversicher ung als ein Teil der Invalidenversicherung macht der kranken Witwe eine Heilstättenkur möglich.

Auch die Unfallversicherung trägt an ihrem Teile zur Bekämpfung der Tuberkulose, die gar nicht selten Folge eines Unfalls ist, bei.

Eine grosse Herabminderung der Tuberkulosesterblichkeit ist entschieden durch die Gewerbehygiene, Einführung der Sonntagsruhe, Beschränkung der Arbeitszeit an Wochentagen (8 Uhr Ladenschluss), durch die Gesetze betreffend Heimarbeit und Beschäftigung Jugendlicher herbeigeführt worden. Die Gewerbeaufsicht des Staates hat in den die Gesundheit stärker angreifenden Betrieben dahin gewirkt, dass Massnahmen zur Verminderung der Schädlichkeiten getroffen wurden. Wenn es auch noch eine ganze Reihe Berufe gibt, die direkt zur Tuberkulose disponieren, so vor allem die der Steinhauer, Glasschleifer, Zigarrenarbeiter usw., so ist zu erwarten, dass auch hier langsam durch weiteren Ausbau die Gesetze Wandel schaffen werden.

\section{Neue Leistungen von Staat und Gemeinde.}

Wie schon in dem vorigen Abschnitt angedeutet wurde, ist uns in Deutschland das Ziel der Tuberkulosebekämpfung, die Beseitigung der Infektionsquelle, etwas aus den Augen entglitten. Wir glaubten lange Zeit mit Einrichtung der Heilanstalten genug getan zu haben. Während England und Amerika durch umfangreiche Bauten

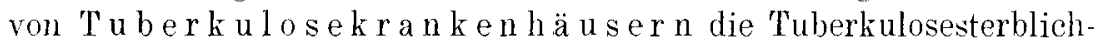
keit zu vermindern suchten und auch einen weiteren Rück. gang erreichten wie wir, ohne das Heer unserer hostbaren Heilanstalten, kommt uns erst in den letzten Jahren alles richtig zum Bewusstscin. Wir verdanken das vor allem der Fürsorgestellenbewegung, die uns auf die grosse Zahl unversorgter Tuberkulöser, die täglich neue Ansteckungsquellen der sie Tmgebenden wurden, aufmerksam machte. Denn nicht nur für die Kinder, sondern auch für die Erwachsenen besteht die Gefahr der Ansteckung. I)arauf weist auch We in berg (l. c.) hin, ebenso Hart (42) und Hillenberg (43). Auch die Infektion von Ehegat ten kommt, wenn auch nicht so häufig, vor.

Die neuen Forderungen bestehen deshalb darin:

A usbau der bestehenden Fürsorgestellen und Schaffen lleuer, besonders a uf dem Lande. Der Ausbau hat nach verschiedenen Richtungen hin zu erfolgen. Die vor- 
nehmste Pflicht der Fürsorgestelle ist es, die Gesunden vor den Kranken zu schützen, andererseits aber auch die Bedrohten oder Leichtkranken heraus zu finden, um die vorbeugenden oder bessernden Massnahmen auszuführen. Die $\mathrm{S}$ ta n de sämter melden die an Tuberkulose Verstorbenen, die Landes-Versicherungsanstalten die in Heilstätten Aufgenommenen, Entlassenen und neuen Invalidenrentner, die Krankenhäuser die aufgenommenen und entlassenen 'Tuberkulösen, die Armenverwaltung, Krankenkassen, Lehrer, 'Pastoren usw. ihnen bekannte Tuberkulosekranke. Die Fürsorgeschwester, auf dem Lande die Gemeindeschwester, besucht die Wohnungen, sicht auf Sauberkeit, vernünftige Platzverteilung, Isolierung, schlägt, wem diese nicht durchführbar ist, die Abgabe eines Bettes oder das Zumieten eines Raumes dem Fürsorgearzt vor und lädt den Kranken und seine Familie zur ärztlichen Untersuchung ein. Der Arzt stellt den Gesundheitszustand fest, veranlasst Krankenhausoder Heilanstaltsaufnahme, für bedrohte Personen Abgabe von Milch, er vermittelt durch die Schwester Essen, lässt den Auswurf untersuchen, gibt Spuckflaschen ab, schlägt Kinder für Ferienkolonien, Walderholungsstätten, Seeaufenthalt vor, und sorgt dafür, dass an Bedürftige, um im Winter auch das Heizen des Isolierraumes durchzuführen, Kohlen abgegeben werden.

Die neuen Wege der Tuberkulosebekämpfung gehen also vor allem dahin: Schutz der Gesunden vor den Kranken. Es wäre unmenschlich, die Kranken das einseitig fühlen zu lassen. Sondem der Kranke soll diesen Zweek kennen, aber andererseits auch erfahren, dass wir dem, der sich willig unseren Vorschlägen fügt, dafür alle Möglichkeiten, seine Krankheit zu bessern, bieten wollen. Da ist in erster Linie das Tuberkulosekrankenhaus, dessen Wirksamkeit eine sehr dankbare ist infolge des Ausbaues der Lungenbehandlung, des Stickstoffverfahrens, der chirurgischen Methoden. Eignet er sich dort nicht, kommt das Pflegeheim, das dem Krankenhaus anzugliedern wäre, in Betracht. Will der Kranke in der Familic bleiben, sorgen wir dafür, dass er ein eigenes Bett erhält, auch ein eigenes Zimmer, für das wir den entsprechenden Zuschuss zur Miete bereitstellen. Tagsüber darf er in die Beschäftigungsstellen und die Liegehallen kommen.

Solange hinreichende und geeignete Möglichkeiten für dic Unterbringung der Tuberkulösen noch nicht zu Gebote stehen, werden wir, um die Schwerkranken im Hause von der Fürsorge für die Familie zu entbinden, Pflegerinnen für die Familien stellen, wo die Kranken nicht selbst mehr für die nötige Sauberkeit sorgen können, Putz- und Waschfrauen geben. Es könnte auffallen, dass 
von der Desinfektion gar nicht gesprochen worden ist. Für noch nötiger als sie halte ich die gründliche regelmässige und häufige Reinigung durch Familienmitglieder oder durch dazu angestellte Putzfrauen. Wir haben in Halle ständig eine Putzfrau, die aufräumen hilft in den Familien der Kranken. Die Einrichtung hat sehr bewährt. Bei Todesfällen ist auch eine gründliche D e s i n f ek tion nötig (s. auch Li ebe l. c.).

Solange der Kranke in der Familie bleibt, ist natürlich eine A ufklärung der Kranken und ihrer Angehörigen mit die Hauptsache. Wir erreichen sie ausser durch die mündlichen Mitteilungen des Arztes und der Schwestern durch Veröffentlichungen in der Presse, regelmässige monatliche Berichte über die Inanspruchnahme der Fürsorgestelle und kurze Arbeiten des Fürsorgearztes. Die hallische Presse z. B. hat unsere kurzen Artikel, wie: „Wie schützen wir uns vor Schwindsucht?" oder ,Vorboten und Anzeichen der Schwindsucht" usw. immer bereitwilligst abgedruckt, ja sie sind vielfach in andere Blätter übergegangen.

Aber die Hauptarbeit der Fürsorgestellen bleibt es, für eine Isolierung und möglichst Asylierung der Kranken zu sorgen. Die Fürsorgestellen sollen möglichst die Kranken aus den Fa milien herausnehmen, für ihre Unterbringung in Krankenhäuser, Pflegeheime usw. sorgen, oder sie in der Familie isolieren; oder die Gesunden herausnehmen aus den Wohn ungen der Kranken, gleicherweise aber Kranke und Gesunde aufklären über die Gefahr der Ansteckung und ihrer Vermeidbarkeit. Bettschirme, wie sie noch vielfach in Fürsorgestellen abgegeben, sind nur ein schlechter Notbehelf. Wäschebeutel für die Wäsche der Kranken, wie wir sie früher viel abgegeben haben, sind nicht unzweckmässig. Schmierseife wird gern und reichlich abgegeben, um nach Kräften die Sauberkeit zu heben. Aber das alles ist gegenüber der Asylierung Stückwerk.

Um die Arbeit der Fürsorgestelle erspriesslich zu gestalten, ist vor allem nötig, dass geeignete Räumlichkeiten, die Kranken aufzunehmen, vorhanden sind. Ungern wird sich der Kranke in Sterbehäuser, Siechenhäuser aufnehmen lassen, die er erfahrungsgemäss nicht mehr lebend verlässt. Gerne aber wird er in heilstättenartig angelegte Heime und Krankenhäuser gehen. Nur soll man ihm, soweit er Rentenempfänger ist, $n$ icht die ganze Rente entziehen, sondern einen Teil der Renteder Familie belassen. Um so eher wird sich der Kranke zu der Übersiedelung entschliessen. Einzelne grosse Städte: so Köln, Berlin, Stettin, Charlottenburg haben bereits eigene 
Tuberkulosekrankenhäuser gebaut für alle Stadien der Krankheit: hier werden Leichtkranke für die Aufnahme in Heilanstalten vorbeobachtet, andere spezifisch behandelt, andere operativ an Kehlkopf und Lunge, andere dauernd asyliert. Die Tuberkulosekrankenhäuser sollen sein wie richtige Krankenhäuser, wie Genesungshäuser: es wird an dem Kranken behandelt, ihm geholfen, er nicht wie sonst so oft in allgemeinen Krankenhäusern als lästige, heilbareren Kranken den Platz raubende Person angesehen. An diese Krankenhäuser wären in den grösseren Städten einerseits $\mathrm{K}$ r i p p e $\mathrm{n}$ mit 'Tag- und Nachtbetrieb anzugliedern, die die Neugeborenen aus tuberkulösen Familien sofort herausnehmen und so vor Ansteckung schützen, andererseits wären Schlafpavillons erforderlich, um die Kinder nachts über aus schlechten Wohnungen fernzuhalten (s. o.); für die offenen Tuberkulösen selbst, soweit sie noch arbeiten können und wollen, sollte man Beschäftigungs s t e ll e n errichten mit Schreibstuben, Gärtnereien, Tischlereien usw., wie das schon an einzelnen Orten geschieht $\_\mathrm{Neubert}(44)^{\urcorner}$. Auf diese Weise ist der Kranke die meiste Zeit des Tages fern von der Familie gehalten und die Quelle der Ansteckung ausgeschaltet. Solche Beschäftigungsplätze können sich natürlich nicht selbst erhalten, sondern brauchen städtische Zuschüsse. Es muss aber für die Städte ratsamer erscheinen, an Stelle der sonst erteilten Armenunterstützung geleistete Arbeit zu bezahlen, wenn sie damit den Kranken eine persönliche Befriedigung. schaffen und die Familie gesund crhalten helfen, durch Fernhaltung des Kranken.

Für schwerere, nicht arbeitende Kranke könnte mit dem Tuberkulosekrankenhaus tagsüber eine Li egehalle ohne Beköstig u 11 g verbunden werden, d. h. der Kranke erhielte nur freie Fahrt auf der elektrischen Bahn und früh und nachmittags je $1 / 2$ Liter Milch; zur Mittagsmahlzeit wäre er nach Hause zu entlassen; will er auf der Liegehalle Mahlzeiten einnehmen, hat er sie mitzubringen.

Kleine Krankenhäuser auf dem Lande sollten mit Liegehallen versehen und mit kleinen Tuberkuloseabteilungen ausgebaut, oder es sollten ihnen kleine Pflegeheime angegliedert werden, wie das im Rheinland und in Westfalen mit Hilfe der Landesversicherungsanstalt geschehen ist (s. Fürst l. c.). Anderswo, wie z. B. in $\mathrm{K} \ddot{o l n}$, hat man eigene $\mathrm{Tuberkulosehäuser}$ in bestimmten Gegenden der Stadt errichtet, mit Balkons zu Liegegelegenheiten. Das halte ich für nicht so günstig, weil so der Kranke in der Familie bleibt.

Man muss scheiden: arbeitende, ausserhalb des Hausestätige Kranke, und solche, die sich nichtausser- 
tätigkeit für Lungenkranke in weitgehendstem Masse der Unterstützung der Stadt.

Die dauernde Herausnahme von $\mathrm{Kindern}$ aus den Familien, wie sie Frankreich im Oeuvre Grancher (s. o.) hat, ist auch von uns ausgeführt worden vom sächsischen Heilstättenverein. In die Kinderkolonie Adelsberg werden Kinder über 6 Jahre, die krank oder gefährdet sind, so lange aufgenommen, bis sie entsprechend gekräftigt sind oder die häuslichen Verhältnisse sich gebessert haben [s. I ckert (37)].

Billiger ist es entschieden, wie wir es auch in $\mathrm{H}$ alle versucht haben, solche Kinder bei gesunden Familien unterzubringen. Auch Breslau hat diese Massnahmen schon in einer grösseren Anzahl von Fällen mit Erfolg getroffen. Nach meiner Meinung müsste diese Massregel in viel grösserem Umfange und viel allgemeiner durchgeführt werden.

Nötig ist, dass auch für die Minderbemittelten geeignete Wohnungen zur Verfügung stehen. Auf die grossen Schäden und Missstände auf diesem Gebiete hat Portm a n n (45) mit Recht hingewiesen. Erforderlichenfalls müssten die Gemeinden für das Bauen geeigneter Wohnungen durch Unterstützung von Baugenossenschaften sorgen. Die Stadt $\mathrm{Halle}$ hat, um den Kleinwohn ung sbau anzuregen und zu unterstützen, folgende Vorkehrungen getroffen: sie gibt an gemeinnützige Bauvereinigungen oder private Untemehmer, die Kleinwohnungsbauten errichten und deren dauernde Benutzung zu diesem Zweck sicher stellen, billigen Grund und Boden, soweit städtisches Baugelände in Betracht kommt, sogar zum Selbstkostenpreise ab. Dann unterstützt sie die Bauenden bei Beschaffung der Grunderwerbs- und Baukapitalien, und zwar durch Bürgschaftsübernahme für Kapitalien bis zu $800_{0}$ der entstanden $€$ Grunderwerbs- und Baukosten, Gewährung von ersten und zweiten Hypotheken, Übernahme von Geschäftsanteilen gemeinnütziges Bauvereinigungen, und durch Begünstigungen der Strassenausbau- und Kanalisationskosten. Natürlich behält sich die Stadt die ständige Kontrolle, Festsetzung der Mietspreise usw. vor. Der Weg wäre auch anderswo gangbar. Meyer-Lierheim. (46) schlägt, statt der dauernden Asylierung, vor, die Kranken in Kol on i en anzusiedeln, und zwar in ganzen Dörfern, mit Geflügel-, Bienenzucht, Obstkultur und Gärtnereien; denn die Asylierung -- darin muss man allerdings beistimmen - wird zumeist unangenehm empfunden. Der Gedanke ist ja schon des öfteren ausgesprochen worden; ob und wie weit er sich durchführen lässt, muss die Zeit lehren. 
In Deutschland gibt es ungefähr $700000 \mathrm{~T}$ u berkulös e. Von ihnen kann nachweislich nur $1 / 10$ in unseren Heilanstalten Aufnahme finden. $9 / 10$ der Kranken leben an ihrem Wohnort in ihrer Familie. Die grösste Zahl von ihnen fällt infolge ihrer Mittellosigkeit der Fürsorgestelle zu. Diese hat also bei weitem das grösste Arbeitsgebiet. Da nimmt es sich wunderbar aus, wenn der Staatsminister Delbrü ck auf der letzten internationalen Tuberkulosekonferenz zu Berlin mitteilen konnte, dass im Jahre 191219 Millionen Mark für $\mathrm{Heilverfahren} \mathrm{wegen} \mathrm{Lungentuberkulose} \mathrm{ausgegeben}$ wurden, aber nur 1/2 Million Mark für laufende Beiträge an A uskunfts - und Fürsorgestellen, an Walderholungsstätten und sonstige Wohlfahrtseinrichtungen aufgewendet wurden. Und dabei erfahren wir aus dem gleichen Munde, dass bei Invalidisierungen, die bis zum 35. Lebensjahre bei Industrie-, Bau- und Bergarbeitern erfolgen, mehr als die Hälfte der Tuberkulose zur Last fallen. Den Heilstätten soll nichts gekürzt werden, aber es muss auch das Notwendige für die Angehörigen der Schwertuberkulösen und für diese selbst nicht versäumt werden.

An den Ortsverwaltungen ist es, ausreichende Mittel, vor allem zum Bau von besonderen Tuberkulosekrankenhäusern, Krippen, Schlafpavillons und Liegehallen zu bewilligen, auch zur Ausgestaltung der Beschäftigungsstelle, zur Abgabe von Mietszuschüssen und Betten. Die Kranken k as sen sind ja nach der neuen Reichsversicherungsordnung in der Lage, von ihren Mitteln solche zur Vorbeugung von Krankheiten zur Verfügung zu stellen. Sie müssten viel reichlicher von dieser Befugnis Gebrauch machen wie bisher.

Die Landesversicherungsanstalten haben sich teilweise schon in den Dienst einer gründlichen Tuberkulosebekämpfung durch Bereitstellung von Isolierhäusern gestellt; so wie erwähnt $\mathrm{R}$ h ein land, Westf al en durch Schaffen von Pflegeheimen, die L.-V.-A. Berlin dadurch, dass sie in Beelitz sogenannte Sanierungsfälle aufnimmt, d. h. Schwertuberkulöse, deren häusliche Verhältnisse ein Herausnehmen des Kranken aus der Wohnung aus Rücksicht auf die Angehörigen im Augenblick notwendig machen (47).

Der Staat könnte uns den Kampf gegen die Tuberkulose sehr erleichtern, wenn er endlich die Anzeigepflicht für offene Tuberkulosen einführte. Wenn wir die Forderung immer wieder und allgemein erheben, werden wir hoffentlich noch einmal die Einführung der Anzeigepflicht erreichen.

Wenn dann auf dem vorgeschlagenen Wege der Kampf von allen Seiten aufgenommen würde, würde er sicher noch weit grössere Ertolge zeitigen und noch mehr dem Worte Geltung verschaffen: „D i e 
Schwindsucht ist eine vermeidbare Krankheit." Dass sie es zurzeit für manche oder viele noch nicht ist, liegt nur an der Unzulänglichkeit der bisher getroffenen Massnahmen. $\mathrm{W}$ ir kennendie Wegezu grösseren Erfolgen, wirmüssen sie nur gehen!

\section{Literatur.}

1. O rth, ther die Bedeutung der Rinderbazillen für den Menschen. Berliner klin. Wochenschr. 1913. Nr. 10.

2. Weber, Die Bedeutung der Rindertuberkelbazillen für den Menschen. Ebenda. Nr. 12. 1913.

3. Derselbe, Die Bedeutung der Rindertuherkulose für die Entstehung der menschlichen Tuberkulose. Zeitschr. für Tub. Bd. 19. H. 6.

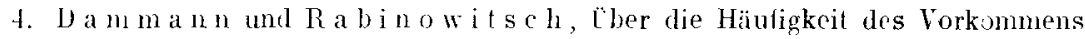
ron Rindertuberkelbazillen beim Menschen. Fibenda. Bd. 21. H. 1-2.

5. Möller, Zur Atiologie ron Knochen- und Gelenktuberkulose. Weutsche med. Wochenschr. 1913. Nr. 38.

6. Besche, Lntersuchungen über die tuberkulöse Infektion in Kindesaller. Ëbenda. 1913. Nr. 10.

7. Schme y, Das Wesen der Skrofulose. Tuberculosis. 1912. Nr. 9.

8. Deist, Beitrag zur Frage der Bedeutung der Perlsuchtbazillen für die Tuberkulose des Kindes. Beitr. zur Klin. der Tub. Bd. 27. H. 3.

9. If a rbitz, ťber angeborene Tulerkulose. Münch. med. Wochenschr. 1913. Nr. 11.

10. v. Leube, l̈ber die Bekämpfung der Tuberkulose in Kindesalter. Tuber. culosis. Nr. 1. 1913.

11. If a $\mathrm{m}$ bu $\mathrm{r}$ g e r, l'ber Tuberkulose des Kindesalters. Med. Klin. 1913. Nr. 13.

12. Kuthy, Cher die Turbansche Vererbung des Locus minoris resistentiae bei Lungentuberkulose. Zeitschr. für Tub. Bd. 20. II. 1.

13. Wolff, Die hämatogene Verbreitung der Tuberkulose und die Disposition bei Tuberkulose. Beitr. z. Klinik der Tub. Bul. 25. Hf. 1.

14. Curschmann, l'ber die Bedeutung der erblichen Belastung für den Verlauf der Lungentuberkulose. Verhandl. der Heilanstaltsïirgte 1912. Kabitzsch, Würzburg.

15. De rselbe, Die erbliche Belastung als pathogenelischer Faktor für die Fntstehung der Lungentuberkulose und ihre Bedentung für den Verlanf der Erkrankung. Tuberculosis. 1914. Nr. 2.

16. Polla k, Das Kind im tuberkulösen Milien. Beitr. zur Klin. der Tub. Bd. 19.

17. B a c meister, Die Freund sche Lehre und der heutige Stand der Frag? von der lokalen Disposition der Lungenphthise, Elenda. Bd. 28. II. 1.

18. Derselbe, Aerogene oder laimatogene Entstehung der Lungenphthise. Deutsche med. Wochenschr. 1913. Nr. 24.

19. Śerog, Zur Disposition der Lungen fur Tuberkulose. Berliner klin. Wochenschr. 1912. Nr. 45. 
20. Schulze, Anomalien des linken Rippenringes und Lungentuberkulose. Beitr. zur Klin. der Tub. Bd. 26. H. 2.

21. $\mathrm{K} \ddot{\mathrm{u}} \mathrm{ch}$ enh off, $\mathrm{Cber}$ die Bedeutung von Wirbelsüulenanomalien für die Entstehung der Lungentuberkulose. Ebenda. Bd. 29. H. 2.

22. Liebe, Die Bedeutung der Ansteckungsgefahr der Heilstätten und für die Bekämpfung der Tuberkulose. Verh. d. Heilanstaltsärzte 1912. Kabitzsch.

23. Blöte, Tuberkuloseimmunität und natürliche Zuchtwahl. Zeitschr. für Tub. Bd. 20. H. 2.

24. De yke, Epidemiologische Beobachtungen über das Auftreten der Tuberkulose in der Türkei. Bericht über die 7. Versamml. der Heilstättenärzte. Kabitzsch, Würzburg.

25. H e i m, Die Tuberkulose in den deutschen Schutzgebieten. Zeitschr. für Tub. Bd. 20. H. 4 .

26. S c he re r, Uber das Vorkommen von Tuberkulose und Syphilis in DeutschSüdwestafrika. Münch. med. Wochenschr. 1913. Nr. 27.

27. B e h l a , Der Verlauf der gesamten Tuberkulosemortalitätsstatistik in Preussen seit 1876 nach Altersklassen und die spezialisierte Kindertuberkulosestatistik. Beriner klin. Wochenschr. 1913. Nr. 42.

28. Diesel, Beitrag zur Statistik der Tuberkulosesterblichkeit in Baden. Deutsche med. Wochenschr. 1913. Nr. 22.

29. Fir $\mathrm{st}$, Welche Vorschläge ergeben sich aus der Verteilung der offenen und geschlossenen Formen von Tuberkalose in und ausserhalb der städtischen Krankenanstalten Münchens für dịe Bekämpfung der Tuberkulose in München? Zeitschr. für Tub. Bd. 21. H. 5.

30. O everland, Untersuchungen mit v. Pirquets Reaktion. Ebenda. Bd. 20. H. 3.

31. Peters, tber den Einfluss der verschiedenen Grade der erblichtuber. kulösen Belastung auf die allgemeine Beschaffenheit der Volksschulkinder. Med. Klin. 1913. Nr. 20.

32. Kruse, Über Verbreitung der Tuberkulose, namentlich in wollhabenden Klassen. Med. Klin. 1913. Nr. 4.

33. D örn e r, Ein Beitrag zur Pathogenese der Tuberkulose. Beitr. zur Klin. der Tub. Bd. 20. H. 1.

34. Weinberg, Die Kinder der Tuberkuiösen. Deutsche med. Wochenschr. 1913. Nr. 28.

35. Engelhardt, Über den Nachweis von Tuberkelbazillen im aspirablen Staub. Beitr, zur Klin. der Tub. Bd. 26. H. 2.

36. Effler, Die Tuberkulosebekämpfung im Säuglingsalter. Erfolge, Mittel und Aussichten. Deutsche med. Wochenschr. 1914. Nr. 7.

37. I ckert, Die Bewahrung der Kinder vor Tuberkulose. Ebenda 1913. Nr. 46.

38. Fischer-Defoy, Ergebnisse über die Lntersuchung der Tuberkuloseverbreitung und die projektierte Tuberkulosebekämpfung im Landkreise Quedlinburg als Muster der Tuberkulosebekämpfung in ländlichen Kreisen. Zeitschr. für Tub. Bd. 20. H. 4-5.

39. F ür $\mathrm{b}$ ring e r, Tuberkuloseuntersuchungen in einem thüringischen Dorf. Beitr. zur Klin. der Tub. Bd. 28. H. 1.

40. Holitscher, Alkoholismus und Tuberkulose. Ebenda. Bd, 29. H. 2.

41. Dörner, Vergleichende Lntersuchung über Tuberkuloseverbreitung in zwei verschiedenen Bezirken Badens. Ebenda. Bd. 30. H. 1.

42. Hart, Beitrag zur Pathologie der Tuberkulose. Med. Klin. 1913. Nr. 50. Beiträge zur Klinık der Tuberknlose. Bd. XXXI, H. 3. 
570 Blümel: Neuere Ansichten über Entstehung u. Verhütung d. Tuberkulose. [30

43. Hillenberg, Epidemiologische Untersuchungen zur Frage der Phthisiogenese. XI. Intern. Tuberkulose-Konferenz.

44. N e u b e r t, C̈ber Arbeitsplätze für tuberkulöse Invalide. Tuberculosis 1914. Nr. 2.

45. Portmann, Tuberkulose und Wohnung. Zeitschr. für Tub. Bd. 20. H. 2.

46. Me yer, Tuberkulose und Isolierung. Deutsche med. Wochenschr. 1913. Nr. 17.

47. Das Tuberkulosefürsorgeverfahren der L.V.A. Berlin. Tuberkulosefürsorgeblatt 1914. Nr. 7. 